\title{
Rashes in Pregnancy
}

\author{
Mark A. Bechtel, MD
}

$\longrightarrow$ ashes that develop during pregnancy often result in considerable anxiety or concern for patients and their families. Recognizing these pregnancy-specific dermatoses is important in identifying fetal risks as well as providing appropriate management and expert guidance for patients regarding future pregnancies. Managing cutaneous manifestations of pregnancy-related disorders is challenging and requires knowledge of potential side effects of therapy for both the mother and fetus. It also is important to appreciate the physiologic cutaneous changes of pregnancy along with their clinical significance and management.

In 2006, Ambrose-Rudolph et $\mathrm{al}^{1}$ proposed reclassification of pregnancy-specific dermatoses, which has since been widely accepted by the academic dermatology community. The 4 most prominent disorders include intrahepatic cholestasis of pregnancy (ICP); pemphigoid gestationis (PG); polymorphic eruption of pregnancy (PEP), also known as pruritic urticarial papules and plaques of pregnancy; and atopic eruption of pregnancy. ${ }^{2}$ It is important to recognize these pregnancy-specific disorders and to understand their clinical significance. The morphology of the eruption as well as the location and timing of the onset of the rash are important clues in making an accurate diagnosis.

\section{Clinical Presentation}

Intrahepatic cholestasis of pregnancy presents with severe generalized pruritus, usually with involvement of the palms and soles, in the late second or third trimester. Pemphigoid gestationis presents with urticarial papules and/or bullae, often in the second or third trimester or postpartum. An important diagnostic clue for PG is involvement near the umbilicus. Polymorphic eruption of pregnancy presents with urticarial papules and plaques; onset occurs in the third trimester or postpartum and initially involves the striae while sparing the umbilicus, unlike in PG. Atopic eruption of pregnancy has an earlier onset than the other pregnancy-specific dermatoses, often in the first or second trimester, and presents with widespread eczematous lesions. ${ }^{3}$

\section{Diagnosis}

The pregnancy dermatoses with the greatest potential for fetal risks are ICP and PG; therefore, it is critical for health care providers to diagnose these dermatoses in a timely manner and initiate appropriate management. Intrahepatic cholestasis of pregnancy is confirmed by elevated serum bile acids (ie, $>10 \mu \mathrm{mol} / \mathrm{L}$ ), often during the third trimester. The risk of fetal morbidity is high in ICP with increased bile acids crossing the placenta causing placental anoxia and impaired cardiomyocyte function. ${ }^{4}$ Fetal risks, including preterm delivery, meconium-stained amniotic fluid, and stillbirth, correlate with the level of bile acids in the serum. ${ }^{5}$ Maternal prognosis is favorable, but there is an increased association with hepatitis $\mathrm{C}$ and hepatobiliary disease. ${ }^{6}$

Diagnosis of PG is confirmed by classic biopsy results and direct immunofluorescence revealing C3 with or without IgG in a linear band along the basement membrane zone. Additionally, complement indirect immunofluorescence reveals circulating IgG anti-basement membrane zone antibodies. Pemphigoid gestationis is associated with increased fetal risks of preterm labor and intrauterine growth retardation. ${ }^{7}$ Clinical findings of PG may present in the fetus upon delivery due to transmission of autoantibodies across the placenta. The symptoms usually are mild. ${ }^{8}$ An increased risk of Graves disease has been reported in mothers with PG.

In most cases, diagnosis of PEP is based on history and morphology, but if the presentation is not classic, skin biopsy must be used to differentiate it from PG as well as more common dermatologic conditions such as contact dermatitis, drug and viral eruptions, and urticaria.

Atopic eruption of pregnancy manifests as widespread eczematous excoriated papules and plaques. Lesions of prurigo nodularis are common.

\section{Comorbidities}

It is important to be aware of specific clinical associations related to pregnancy-specific dermatoses. Pemphigoid gestationis has been associated with gestational trophoblastic tumors including hydatiform mole and choriocarcinoma. ${ }^{4}$ An increased risk for Graves disease has been reported 
in patients with PG. ${ }^{9}$ Patients who develop ICP have a higher incidence of hepatitis C, postpartum cholecystitis, gallstones, and nonalcoholic cirrhosis. ${ }^{8}$ Polymorphic eruption of pregnancy is associated with a notably higher incidence in multiple gestation pregnancies. ${ }^{2}$

\section{Treatment and Management}

Management of ICP requires an accurate and timely diagnosis, and advanced neonatal-obstetric management is critical. ${ }^{3}$ Ursodeoxycholic acid is the treatment of choice and reduces pruritus, prolongs pregnancy, and reduces fetal risk. ${ }^{4}$ Most stillbirths cluster at the 38th week of pregnancy, and patients with ICP and highly elevated serum bile acids $(>40 \mu \mathrm{mol} / \mathrm{L})$ should be considered for delivery at 37 weeks or earlier. ${ }^{5}$

Management of the other cutaneous disorders of pregnancy can be challenging for health care providers based on safety concerns for the fetus. Although it is important to minimize risks to the fetus, it also is important to adequately treat the mother's cutaneous disease, which requires a solid knowledge of drug safety during pregnancy. The former US Food and Drug Administration classification system using A, B, C, D, and X pregnancy categories was replaced by the Pregnancy Lactation Label Final Rule, which provides counseling on medication safety during pregnancy..$^{10}$ In 2014, Murase et $\mathrm{al}^{11}$ published a review of dermatologic medication safety during pregnancy, which serves as an excellent guide.

Before instituting treatment, the therapeutic plan should be discussed with the physician managing the patient's pregnancy. In general, topical steroids are considered safe during pregnancy, and low-potency to moderate-potency topical steroids are preferred. If possible, use of topical steroids should be limited to less than $300 \mathrm{~g}$ for the duration of the pregnancy. Fluticasone propionate should be avoided during pregnancy because it is not metabolized by the placenta. When systemic steroids are considered appropriate for management during pregnancy, nonhalogenated corticosteroids such as prednisone and prednisolone are preferred because they are enzymatically inactivated by the placenta, which results in a favorable maternal-fetal gradient. ${ }^{12}$ There has been concern expressed in the medical literature that systemic steroids during the first trimester may increase the risk of cleft lip and cleft palate.,12 When managing pregnancy dermatoses, consideration should be given to keep prednisone exposure below $20 \mathrm{mg} / \mathrm{d}$, and try to limit prolonged use to $7.5 \mathrm{mg} / \mathrm{d}$. However, this may not be possible in PG. ${ }^{3}$ Vitamin D and calcium supplementation may be appropriate when patients are on prolonged systemic steroids to control disease.

Antihistamines can be used to control pruritus complicating pregnancy-associated dermatoses. Firstgeneration antihistamines such as chlorpheniramine and diphenhydramine are preferred due to long-term safety data. ${ }^{3,11,12}$ Loratadine is the first choice and cetirizine is the second choice if a second-generation antihistamine is preferred. ${ }^{3}$ Loratadine is preferred during breastfeeding due to less sedation. ${ }^{12}$ High-dose antihistamines prior to delivery may cause concerns for potential side effects in the newborn, including tremulousness, irritability, and poor feeding.

\section{Recurrence}

Women with pregnancy dermatoses often are concerned about recurrence with future pregnancies. Pemphigoid gestationis may flare with subsequent pregnancies, subsequent menses, or with oral contraceptive use. ${ }^{3}$ Recurrence of PEP in subsequent pregnancies is rare and usually is less severe than the primary eruption. ${ }^{8}$ Often, the rare recurrent eruption of PEP is associated with multigestational pregnancies. ${ }^{2}$ Mothers can anticipate a recurrence of ICP in up to $60 \%$ to $70 \%$ of future pregnancies. Patients with AEP have an underlying atopic diathesis, and recurrence in future pregnancies is not uncommon. ${ }^{8}$

\section{Final Thoughts}

In summary, it is important for health care providers to recognize the specific cutaneous disorders of pregnancy and their potential fetal complications. The anatomical location of onset of the dermatosis and timing of onset during pregnancy can give important clues. Appropriate management, especially with ICP, can minimize fetal complications. A fundamental knowledge of medication safety and management during pregnancy is essential. Rashes during pregnancy can cause anxiety in the mother and family and require support, comfort, and guidance.

\section{REFERENCES}

1. Ambrose-Rudolph CM, Müllegger RR, Vaughn-Jones SA, et al. The specific dermatoses of pregnancy revisited and reclassified: results of a retrospective two-center study on 505 pregnant patients. J Am Acad Dermatol. 2006;54:395-404.

2. Bechtel M, Plotner A. Dermatoses of pregnancy. Clin Obstet Gynecol. 2015;58:104-111.

3. Bechtel M. Pruritus in pregnancy and its management. Dermatol Clin. 2018;36:259-265

4. Ambrose-Rudolph CM. Dermatoses of pregnancy-clues to diagnosis, fetal risk, and therapy. Ann Dermatol. 2011;23:265-275.

5. Geenes V, Chappell LC, Seed PT, et al. Association of severe intrahepatic cholestasis of pregnancy with adverse pregnancy outcomes: a prospective population-based case-controlled study. Hepatology. 2014;59:1482-1491.

6. Bergman H, Melamed N, Koven G. Pruritus in pregnancy: treatment of dermatoses unique to pregnancy. Can Fam Physician. 2013;59:1290-1294.

7. Beard MP, Millington GW. Recent developments in the specific dermatoses of pregnancy. Clin Exp Dermatol. 2012;37:1-14.

8. Shears S, Blaszczak A, Kaffenberger J. Pregnancy dermatosis. In: Tyler KH, ed. Cutaneous Disorders of Pregnancy. 1st ed. Springer Nature; 2020:13-39.

9. Lehrhoff S, Pomeranz MK. Specific dermatoses of pregnancy and their treatment. Dermatol Ther. 2015;26:274-284.

10. Content and format of labeling for human prescription drug and biological products; requirements for pregnancy and lactation labeling. Fed Registr. 2014;79:72064-72103. To be codified at 21 CFR § 201.

11. Murase JE, Heller MM, Butler DC. Safety of dermatologic medications in pregnancy and lactation: part 1. pregnancy. J Am Acad Dermatol. 2014;401:E1-E14.

12. Friedman B, Bercovitch L. Atopic dermatitis in pregnancy. In: Tyler KH, ed. Cutaneous Disorders of Pregnancy. Springer Nature; 2020:59-74. 